\title{
A method of fundamental solution without fictitious boundary
}

\author{
W. Chen \& F. Z. Wang \\ Center for Numerical Simulation Software in Engineering and Sciences, \\ Department of Engineering Mechanics, Hohai University, China
}

\begin{abstract}
This paper proposes a novel meshless boundary method called the singular boundary method (SBM). This method is mathematically simple, easy-to-program, and truly meshless. Like the method of fundamental solution (MFS), the SBM employs the singular fundamental solution of the governing equation of interest as the interpolation basis function. However, unlike the MFS, the source and collocation points of the SBM coincide on the physical boundary without the requirement of fictitious boundary. In order to avoid the singularity at origin, this method proposes an inverse interpolation technique to evaluate the singular diagonal elements of the interpolation matrix. This study tests the SBM successfully to three benchmark problems, which shows that the method has rapid convergent rate and is numerically stable.

Keywords: singular boundary method, singular fundamental solution, inverse interpolation technique, singularity at origin.
\end{abstract}

\section{Introduction}

Meshless methods and their applications have attracted huge attention in recent decades, since methods of this type avoid the perplexing mesh-generation in the traditional mesh-based methods such as the finite element method and the finite difference method. In comparison with the boundary element method, a variety of boundary-type meshless methods have been developed. For instance, the method of fundamental solutions (MFS) [1-4], boundary knot method [5], boundary collocation method [6], boundary node method [7,8], regularized meshless method (RMM) $[9,10]$, and modified method of fundamental solution [11] etc.

Since the boundary node method still requires meshes in its numerical integration, it is not a truly meshless scheme like those moving least square based meshless finite element method [12]. 
The MFS is an attractive method with merits being integration-free, truly meshless, super-convergent, and easy-to-use. On the downside, the MFS requires fictitious boundaries outside the physical domain to avoid the singularity at origin because of its use of singular fundamental solution of the governing equation. In practical applications, this artificial boundary is somewhat arbitrary and not easy to determine optimally and makes the MFS unstable in the solution of complexshaped boundary problems.

As an alternative method, the BKM [5] has been introduced to use the nonsingular kernel functions such as general solutions or T-complete functions [13] which also satisfy the governing equation. The collocation and source points are coincident and are placed on the physical boundary of the problem in the BKM. However, the nonsingular kernel functions are not available in some important cases such as Laplace equation.

The RMM has recently been introduced by Young et al. [9]. This method has advantages over the above-mentioned meshless boundary methods in that it applies the desingularization of subtracting and adding-back technique to regularize the singularity and hypersingularity of its interpolation basis function, namely, doublelayer potential. With the increasing of the boundary knot number, the condition number of this method remains small and almost not changed. However, our numerical experiments find that the solution accuracy and the convergence rate of the RMM are not very accurate. In addition, the method requires the equally spaced points to guarantee the solution accuracy and stability, which seriously restricts its applicability to the real-world problems. Based on an idea similar to the RMM, Božidar Šarler [11] proposes the modified method of fundamental solution (MMFS), which has better accuracy than the RMM, but the MMFS method requires numerical integration.

In this study, we propose a novel meshless boundary method called the singular boundary method (SBM) [14]. This method is mathematically simple, accurate, easy-to-program, and truly meshless. Similar to the MMFS, the SBM also directly uses the singular fundamental solution of governing equation of interest as the interpolation basis function. Dissimilar to the MMFS and all other boundary-type meshless methods, the SBM uses an inverse interpolation technique to evaluate the diagonal elements of the interpolation matrix to circumvent the singularity at origin of fundamental solutions. In the rest part of this paper, numerical experiments of this method are presented to demonstrate its convergence, accuracy and stability.

\section{Formulation of singular boundary method}

Without loss of generality, we consider the Laplace equation boundary value problems as described below

$$
\begin{gathered}
\Delta u(x)=0 \quad \text { in } \Omega \\
u(x)=\bar{u}(x) \quad \text { on } \Gamma_{1} \\
m(x)=\bar{m}(x) \quad \text { on } \Gamma_{2}
\end{gathered}
$$


where $u(x)$ and $m(x)=\frac{\partial u(x)}{\partial n}$ are the potential and its normal derivative (flux), respectively, $n$ denotes the unit outward normal, $\Omega$ is the physical solution domain in $\mathcal{R}^{d}$, where $d$ denotes the dimensionality of the space, and $\partial \Omega=\Gamma_{1} \cup \Gamma_{2}$ represents its boundary. For the two dimensional Laplace equation, the fundamental solution is given by

$$
u_{L}^{*}=\frac{1}{2 \pi} \ln \left\|x_{i}-x_{j}\right\| .
$$

The approximate representation of the MFS solution for this problem is written as $[3,15-17]$

$$
u\left(x_{i}\right)=\sum_{j=1}^{N} \nu_{j} \ln \left\|x_{i}-x_{j}\right\|
$$

where $N$ represents the total number of boundary collocation points, $\nu_{j}$ are the unknown coefficients. Obviously, the superposition of the collocation points $x_{i}$ and source points $x_{j}$ will lead to the well-known singularity at origin, namely, $\ln \left\|x_{i}-x_{j}\right\|=\ln 0$ for $i=j$. In order to circumvent this troublesome problem, the MFS places the source nodes on the fictitious boundary outside the physical domain. However, despite of great effort of 40 years, the placement of fictitious boundary in the MFS remains a perplexing problem for complex-shaped boundary problems.

Like the MFS, the SBM also uses the fundamental solution as the kernel function of approximation. Unlike the MFS, the collocation and source points of the SBM are coincident and are placed on the physical boundary without the need of fictitious boundary. The interpolation formula of the SBM is given by

$$
u\left(x_{i}\right)=\sum_{j=1, j \neq i}^{N} \alpha_{j} \ln \left\|x_{i}-x_{j}\right\|+\alpha_{i} q_{i i}
$$

where $\alpha_{j}$ are the unknown coefficients, $q_{i i}$ are defined as the origin intensity factor. Eqn (6) of the SBM differs from eqn (5) of the MFS in that the fundamental solution at origin is replaced by $q_{i i}$ when the collocation point $x_{i}$ and source point $x_{j}$ coincide $(i=j)$.

The MMFS [4] also uses the fundamental solution as the interpolation basis function while placing the source and collocation nodes at the same physical domain. The essential difference between the SBM and MMFS is how to evaluate the origin intensity factor $q_{i i}$. The latter uses the numerical integration approach, while the SBM develops an inverse interpolation technique as detailed below to calculate $q_{i i}$.

The matrix form of equation (6) can be written as

$$
\left\{q_{i j}\right\}\left\{\alpha_{j}\right\}=\left\{u\left(x_{i}\right)\right\}
$$

where $q_{i j}=\ln \left\|x_{i}-x_{j}\right\|$. We can see that $q_{i i}$ are actually the diagonal elements of matrix $Q=\left\{q_{i j}\right\}$. By collocating $N$ source points on physical boundary to satisfy 
the Dirichlet boundary condition eqn (2) and the Neumann boundary condition eqn (3), we obtain the following discretization algebraic equations

$$
\begin{aligned}
\sum_{j=1}^{N_{1}} \alpha_{j} \ln \left\|x_{i}-x_{j}\right\| & =\bar{u}\left(x_{i}\right), \quad x_{i} \in \Gamma_{1}, \\
\sum_{j=N_{1}+1}^{N} \alpha_{j} \frac{\partial \ln \left\|x_{i}-x_{j}\right\|}{\partial n} & =\bar{m}\left(x_{i}\right), \quad x_{i} \in \Gamma_{2},
\end{aligned}
$$

where $N_{1}$ denotes the number of source points placed on the Dirichlet boundary. Obviously, we can not simply use the fundamental solutions to compute $q_{i i}$. Instead we propose an inverse interpolation technique (IIT) to evaluate the diagonal elements $q_{i i}$ of interpolation matrix $Q$ in the SBM.

For the boundary value problem eqns (1)-(3), we locate source points $x_{j}$ on the physical boundary and place computational collocation points $x_{k}$ inside physical domain. And then, we use a simple particular solution as the sample solution of Laplace equation (1), for example, $u=x+y$. Using the interpolation formula eqn (6), we can get

$$
\left\{b_{j k}\right\}\left\{s_{j}\right\}=\left\{x_{k}+y_{k}\right\},
$$

where $b_{j k}=\ln \left\|x_{k}-x_{j}\right\|$. Thus, the influence coefficients $s_{j}$ can be evaluated.

Replacing the computational collocation points $x_{k}$ with the boundary source points $x_{j}$, we have

$$
\left\{q_{j k}\right\}\left\{s_{j}\right\}=\left\{x_{j}+y_{j}\right\},
$$

where the off-diagonal elements of interpolation matrix $Q=\left\{q_{j k}\right\}$ can be computed by $q_{j k}=\ln \left\|x_{k}-x_{j}\right\|$. It is noted that the influence coefficients of the eqn (10) are the same as in eqn (11). Therefore, eqn (11) can be solved to calculate the unknown diagonal elements $q_{i i}$ of the matrix $Q$.

With the previously-calculated origin intensity factor, the SBM can be used to compute arbitrary Laplace problems with the same geometry by using interpolation formula eqn (6). Here we summarize the SBM solution procedure by the following two steps:

Step 1. First, we place source points $x_{j}$ on the physical boundary and select computational collocation points $x_{k}$ inside physical domain. And then we choose a simple sample solution of the governing equation of interest and us the SBM interpolation formula to get its physical solutions at these interior nodes. Since the sample solution is known, we can calculate the influence coefficients $s_{j}$ of the boundary source points $x_{j}$. Finally, replacing the computational collocation points $x_{k}$ by the boundary source points $x_{j}$, we can calculate the origin intensity factor $q_{i i}$.

Step 2. Using the origin intensity factor evaluated from Step 1 and the interpolation formula (6), we can solve arbitrary problems with the same geometry and governing equation. 
As discussed in the following section 3, the diagonal elements of Laplace equation with circular physical domain do not need to use the inverse interpolation technique to evaluate numerically. They are simply a summation of the corresponding off-diagonal elements, that is,

$$
Q(i, i)=\sum_{i \neq j, j=1}^{N} q(i, j) .
$$

However, this is an very exceptional case. To our numerical experiments, the inverse interpolation technique has to be used to determine the diagonal elements in all other geometric domains, irrespective of domain regularity and nodes placement.

\section{Numerical results and discussions}

In this section, based on the above-mentioned numerical formulation, we examine three benchmark examples. The relative average error(root mean square relative error: RMSRE) is defined as follows [18]:

$$
\operatorname{RMSRE}=\sqrt{\frac{1}{K} \sum_{j=1}^{K} \operatorname{Rerr}^{2}}
$$

where Rerr $=\left|\frac{u\left(x_{j}\right)-\tilde{u}\left(x_{j}\right)}{u\left(x_{j}\right)}\right|$ for $\left|u\left(x_{j}\right)\right| \geq 10^{-3}$ and Rerr $=\left|u\left(x_{j}\right)-\tilde{u}\left(x_{j}\right)\right|$, for $\left|u\left(x_{j}\right)\right|<10^{-3}$, respectively, $j$ is the index of inner point of interest, $u\left(x_{j}\right)$ and $\bar{u}\left(x_{j}\right)$ denote the analytical and numerical solutions at the $j$-th inner point, respectively, and $K$ represents the total number of test points of interest.

Unless otherwise stated, the diagonal elements of interpolation matrices in the following three cases are all numerically evaluated by using the inverse interpolation technique. For Laplace governing equation, we use $u=x+y$ as the known sample solution; for Helmholtz equation

$$
\Delta u(x)+\lambda^{2} u(x)=0,
$$

$u=\sin (x) \cos (y)$ is chosen as the known sample solution, where $\lambda$ represents the wave number.

\subsection{Case 1: Circular domain case}

For convenience, the boundary points are distributed uniformly on a unit circle. The exact solution of this case is $u=x^{2}-y^{2}$. To examine the resulting solution accuracy, the number of testing points scattered over the region of interest is chosen to be 620 . 
Here, the diagonal elements of the SBM interpolation matrix are evaluated by two different approaches: (1) eqn (13), a summation of the corresponding offdiagonal elements and (2) the inverse interpolation technique introduced in Section 2 .

The relative average error versus boundary point numbers for this problem is illustrated in fig. 1. It is noted that the SBM error curves using the approach of a summation of the corresponding off-diagonal elements (called the summation approach in fig. 1) and the inverse interpolation technique for the diagonal elements are very close. To our experimental experiences, it is stressed that a summation of the corresponding off-diagonal elements to evaluate diagonal elements only works for the circular domain Laplace problems. On the other hand, it is observed that error curves of both the SBM and the RMM are decreasing with increasing boundary points, while the SBM converges faster than the RMM. When the boundary point number $K=100$, the SBM solution accuracy is of order $10^{-5}$, which is three orders of magnitude less than the RMM one $10^{-2}$.

Figures 2 and 3 show the condition numbers of the RMM and the SBM against the boundary point numbers, respectively. We can see that the RMM condition number is relatively smaller than the SBM ones, which may be an attractive advantage in solving large-scale problems.

\subsection{Case 2: Multiply connected domain case}

Here we consider a multiply connected domain case. The geometric configuration is depicted in fig. 4 , and the exact solution is $u=x^{2}-y^{2}+x y$. The number of testing points scattered across the connected domain is 651 .

Figure 5 shows the convergence rate of relative average error versus boundary point numbers, which indicates that the SBM has a faster convergence rate than the RMM. We also observe that SBM convergence curves oscillates, while the RMM has a stable convergence. The reason for the SBM oscillatory convergence curve may be due to the severely ill-conditioned interpolation matrix. On the other hand, the solution accuracy of the SBM solution appears higher than that of the RMM.

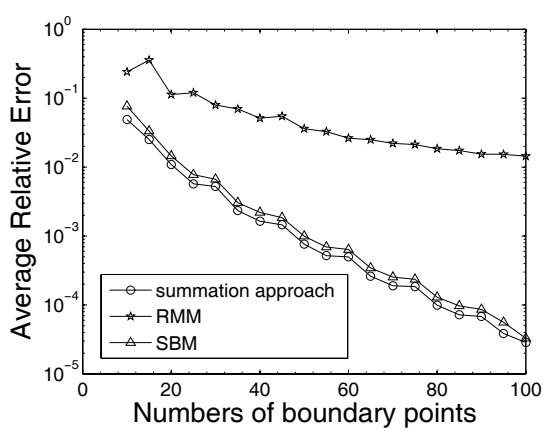

Figure 1: Relative average error curves for Case 1. 


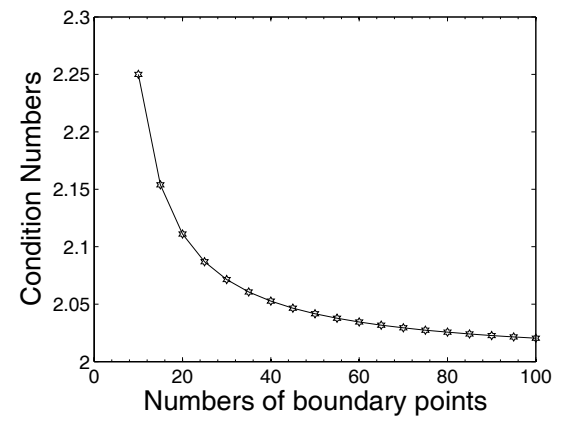

Figure 2: Condition number curve for Case 1 by using RMM.

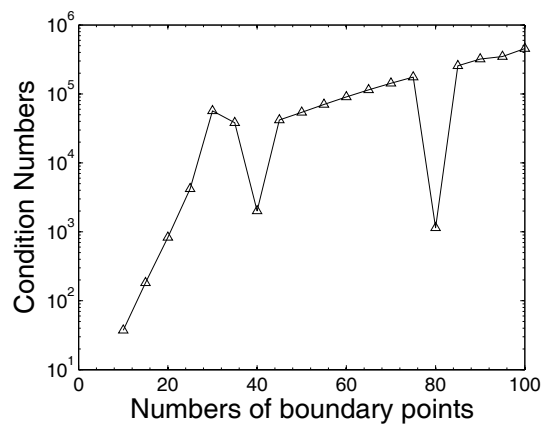

Figure 3: Condition number curve for Case 1 by using SBM.

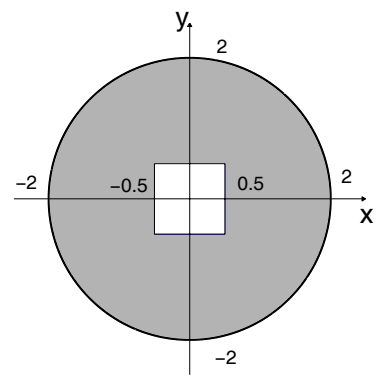

Figure 4: Configuration of 2D multiply connected domain.

For example, the SBM relative average error is $\mathrm{RMSRE}=3.734 \times 10^{-4}$ with boundary point number $K=200$, in contrast to $\mathrm{RMSRE}=1.069 \times 10^{-1}$ for the RMM. 


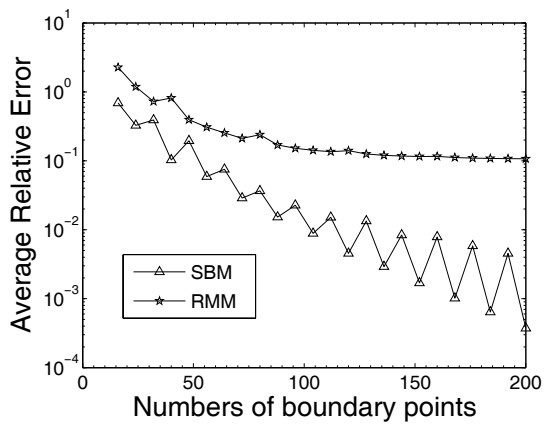

Figure 5: Relative average error curves for Case 3.

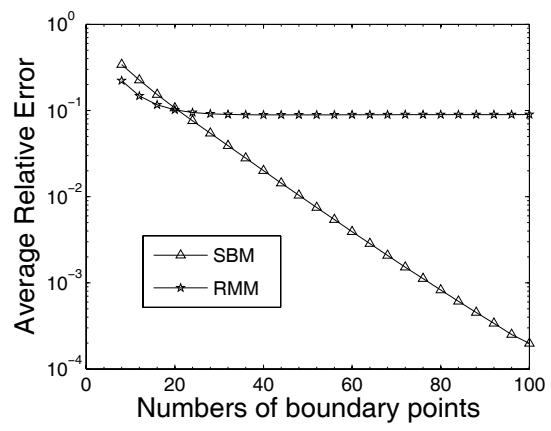

Figure 6: Relative average error curves for Case 4.

\subsection{Case 3: Helmholtz equation case}

Besides the previous Laplace equation cases, we also examine Helmholtz problems with wave number $k=\sqrt{2}$ and its exact solution is $u=\sin (x+0.5) \cos (y)$. The boundary points are distributed uniformly on a unit square domain. We choose 1369 test points scattered across the physical domain.

Figures 6 and 7 show the relative average errors and the condition numbers versus boundary point numbers of the SBM and the RMM, respectively. Unlike the previous two Laplacian cases, we can see that the RMM accuracy is better than the SBM one when the boundary point number $K \leq 20$. But with the increasing number of boundary points, the relative average error of the SBM improves faster and exhibits a rapid convergence rate, while the relative average error of the RMM remains RMSRE $=1.0 \times 10^{-1}$.

Figure 7 shows that the condition numbers of the RMM are relatively lower than those of the SBM. For both methods, the condition numbers are no more than $10^{2}$. 


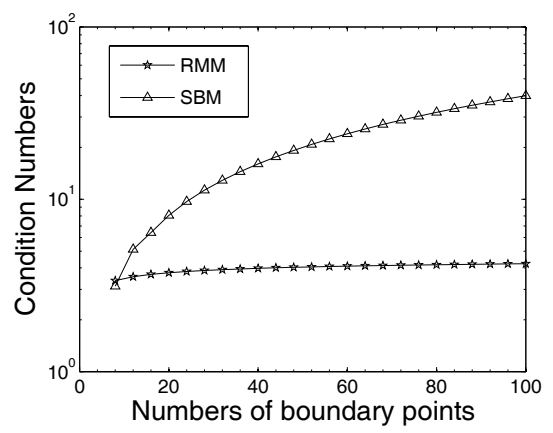

Figure 7: Condition number curves for Case 4.

\section{Conclusions}

This paper introduces a novel meshless singular boundary method. Like the MFS, the RMM and the MMFS, the SBM uses the fundamental solution as the interpolation basis function. Unlike the MFS, the source and collocation points coincide in the present method and the fictitious boundary in the MFS is no longer required. Also, unlike the RMM and the MMFS, the SBM uses a new inverse interpolation technique to remedy the singularity at origin of the fundamental solutions. Numerical solutions of the SBM agree well with the analytical solutions. From the foregoing figures of relative average error versus the increasing number of boundary points, we can see that numerical results of both the SBM and the RMM exhibit stable convergence trend in all tested cases, while the SBM converges faster than the RMM. However, it is also observed that the RMM condition number is in general much smaller than the SBM one in all tested cases.

Mathematical analysis of the SBM is now still under study and will be reported in a subsequent paper.

\section{Acknowledgement}

The work described in this paper was supported by a research project funded by the National Natural Science Foundation of China (Project No. 10672051).

\section{References}

[1] Bogomolny, A., Fundamental solutions method for elliptic boundary value problems. SIAM Journal on Numerical Analysis, 22, pp. 644-69, 1985.

[2] Fairweather, G. \& Karageorghis, A., The method of fundamental solutions for elliptic boundary value problems, Advances in Computational Mathematics, 9, pp. 69-95, 1998. 
[3] Golberg, M.A. \& Chen, C.S., The method of fundamental solutions for potential, Helmholtz and diffusion problems, In: Golberg MA, editor. Boundary integral methodsłnumerical and mathematical aspects. Southampton: Computational Mechanics Publications; pp. 103-76, 1998.

[4] Chen, C.S., Hokwon A. Cho. \& Golberg, M.A., Some comments on the illconditioning of the method of fundamental solutions, Engineering Analysis with Boundary Elements, 30, pp. 405-410, 2006.

[5] Chen, W. \& Hon, Y.C., Numerical investigation on convergence of boundary knot method in the analysis of homogeneous Helmholtz, modified Helmholtz and convection-diffusion problems, Computer Methods in Applied Mechanics and Engineering, 192, pp. 1859-1875, 2003.

[6] Chen, J.T., Chen, I.L., Chen, K.H. \& Y.T. Yeh. A meshless method for free vibration of arbitrarily shaped plates with clamped boundaries using radial basis function, Engineering Analysis with Boundary Elements, 28, pp. 535545, 2004.

[7] Mukherjee, Y.X. \& Mukherjee, S., The boundary node method for potential problems, International Journal for Numerical Methods in Engineering, 40, pp. 797-815, 1997.

[8] Zhang, J.M., Yao, Z.H. \& Li, H., A hybrid boundary node method, International Journal for Numerical Methods in Engineering, 2002;53:751-763.

[9] Young, D.L., Chen, K.H. \& Lee, C.W., Novel meshless method for solving the potential problems with arbitrary domains, Journal of Computational Physics, 209, pp. 290-321, 2005.

[10] Young, D.L., Chen, K.H., Chen, J.T. \& Kao, J.H., A Modified Method of Fundamental Solutions with Source on the Boundary for Solving Laplace Equations with Circular and Arbitrary Domains, CMES: Computer Modeling in Engineering and Science, 19(3), pp. 197-221, 2007.

[11] Božidar, Ś. A modified method of fundamental solutions for potential problem, In: Chen, C.S., Karageorghis, A. \& Smyrlis, Y.S., editor. The Method of Fundamental Solutions - A Meshless Method, Dynamic Publishers, pp. 299321, 2008.

[12] Atluri, S.N. \& Zhu, T., New concepts in meshless methods, International Journal for Numerical Methods in Engineering, 47, pp. 537-556, 2000.

[13] Kita, E., Kamiya, N. \& Ikeda, Y., An application of Trefftz method to the sensitivity analysis of two-dimensional potential problem. International Journal for Numerical Methods in Engineering, 38, pp. 2209-2224, 1995.

[14] Chen, W., Singular boundary method: A novel, simple, meshfree, boundary collocation numerical method. Acta Mechanica Solida Sinica, (in Chinese)(acceptted).

[15] Barnett, A.H. \& Betcke, T., Stability and convergence of the method of fundamental solutions for Helmholtz problems on analytic domains. Journal of Computational Physics, 227, pp. 7003-7026, 2008.

[16] Wei, T., Hon, Y.C. \& Ling, L., Method of fundamental solutions with regularization techniques for Cauchy problems of elliptic operators, Engineering Analysis with Boundary Elements, 31, pp. 373-385, 2007. 
[17] Johansson, B.T. \& Lesnic, D., A method of fundamental solutions for transient heat conduction, Engineering Analysis with Boundary Elements, 32, pp. 697-703, 2008.

[18] Wang, F.Z., Chen, W. \& Jiang, X.R., Investigation of regularized techniques for boundary knot method, Communications in Numerical Methods in Engineering, (acceptted). 